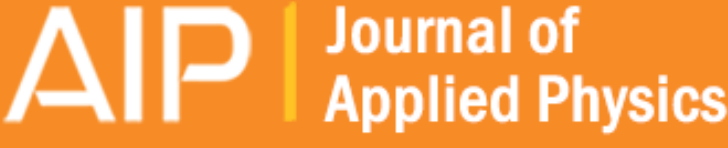

\section{On a point defect inside an idealized elastic sphere}

Chih-Bing Ling and Charles R. Houska

Citation: Journal of Applied Physics 54, 5123 (1983); doi: 10.1063/1.332734

View online: http://dx.doi.org/10.1063/1.332734

View Table of Contents: http://scitation.aip.org/content/aip/journal/jap/54/9?ver=pdfcov

Published by the AIP Publishing

\section{Articles you may be interested in}

Elastic wave propagation in a solid layer with laser-induced point defects

J. Appl. Phys. 110, 064906 (2011); 10.1063/1.3633524

Magnetohydrodynamic activity inside a sphere

Phys. Fluids 18, 116602 (2006); 10.1063/1.2393438

Elastic interaction between a point defect and an edge dislocation

J. Appl. Phys. 50, 1263 (1979); 10.1063/1.326155

Elastic Field of a Point Defect in a Cubic Medium and its Interaction with Defects

J. Appl. Phys. 41, 3930 (1970); 10.1063/1.1658391

Interactions of Point Defects and Elastic Inclusions

J. Appl. Phys. 38, 595 (1967); 10.1063/1.1709380

MIT LINCOLN

LABORATORY CAREERS

Discover the satisfaction of innovation and service

to the nation
- Space Control

- Air \& Missile Defense

- Communications Systems \& Cyber Security

- Intelligence, Surveillance and Reconnaissance Systems

- Advanced
Electronics
- Tactical Systems
- Homeland
Protection
- Air Traffic Control

- Air Traffic Control

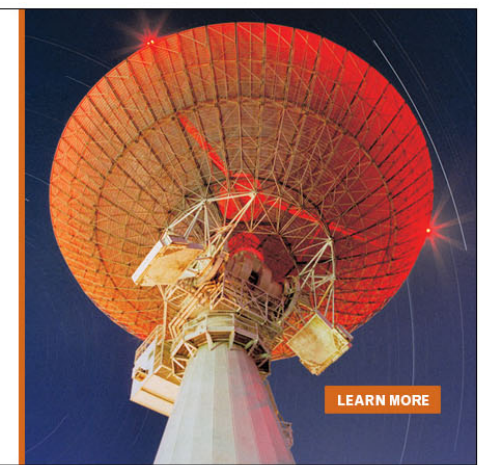




\title{
On a point defect inside an idealized elastic sphere
}

\author{
Chih-Bing Ling ${ }^{\text {a) }}$ and Charles R. Houska ${ }^{\text {b) }}$ \\ Virginia Polytechnic Institute and State University, Blacksburg, Virginia
}

(Received 12 July 1982; accepted for publication 25 April 1983)

This paper presents a method of solution for the displacement, stress, and strain due to a point defect located inside a sphere. The solution is represented by a Love stress function in spherical coordinates, which is biharmonic in character. Two axisymmetric types of the point defect are considered. One is treated as a center of dilatation and the other as a double force without moment, or a doublet, oriented axisymmetrically. The Love stress function for the point defect in an infinite solid is specified in each case by a single biharmonic function. The residual tractions on the surface of the sphere left by this function are annulled by superposing two series of biharmonic functions. When the Love stress function is determined, the displacement, stress, and strain can be derived straightforwardly.

PACS numbers: 71.55. - i, 61:70.Ey

\section{INTRODUCTION}

A point defect in a crystalline solid is usually treated as a center of dilatation ${ }^{1,2}$ when it is spherically symmetric. The anisotropy of the solid is neglected in the sense that the solid is idealized as a homogeneous isotropic elastic medium. Such a treatment is satisfactory only when the solid is of infinite size. When it is of finite size or at least one of its dimensions is finite, residual tractions are left on its boundary. Such tractions vanish identically on a boundary located at infinity. In 1954, Eshelby ${ }^{3}$ proposed to introduce an additional system of negative tractions to annul the residual tractions.

The determination of displacement or stress in a solid directly by integration when only the surface tractions are known generally presents mathematical difficulties. This was mentioned by Eshelby himself and also later by Dundurs and Guell. ${ }^{4}$ The displacement, stress, and also the strain in the solid can be derived from one or more sets of biharmonic functions appropriate to the problem. Several approaches are available to achieve the purpose. The solution is considerably simplified whenever the problem possesses an axis of symmetry.

A center of dilatation is by nature a spherically symmetric elastic singularity, which can be resolved into three mutually orthogonal double forces without moment of equal strengths. Since each double force without moment can be treated separately, it is possible to construct a point defect of unequal strengths in orthogonal directions. For brevity, a double force without moment will henceforth be described as a doublet.

This paper presents a method of solution for the displacement, stress, and strain due to a point defect located inside an idealized sphere. Two types of the point defect are considered. One is treated as a center of dilatation and the other as a doublet oriented axisymmetrically. Both types possess an axis of symmetry. Love's theory of symmetric strain $^{5}$ is adapted in the solution and is represented by a biharmonic function commonly called a Love stress func-

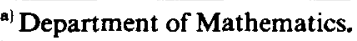

${ }^{b}$ Department of Materials Engineering. tion. When such a function is determined, the displacement, stress, and strain in the sphere can be derived straightforwardly.

\section{COORDINATES AND FORMULAS}

Define a set of spherical coordinates $(\rho, \phi, \theta)$ by

$$
\begin{aligned}
& x=\rho \sin \phi \cos \theta, \\
& y=\rho \sin \phi \sin \theta, \\
& z=\rho \cos \theta,
\end{aligned}
$$

where $(x, y, z)$ is a set of cartesian coordinates with its origin at 0 . Also let

$$
\mu=\cos \phi .
$$

In the axisymmetric case with the $z$ axis as an axis of symmetry, the Love stress function is independent of $\theta$. The formulas for the components of displacement, stress, and strain referred to spherical coordinates were given by Ling and Yang $^{6}$ some time ago. They are as follows:

(i) For the components of displacement,

$$
\begin{aligned}
u_{\rho}= & \frac{1}{2 G}\left[2(1-v) \mu \nabla^{2}-\frac{\partial}{\partial \rho}\left(\mu \frac{\partial}{\partial \rho}+\frac{1-\mu^{2}}{\rho} \frac{\partial}{\partial \mu}\right)\right] \chi \\
u_{\phi}= & \frac{1}{2 G}\left(1-\mu^{2}\right)^{1 / 2}\left[-2(1-v) \nabla^{2}\right. \\
& \left.+\frac{1}{\rho} \frac{\partial}{\partial \mu}\left(\mu \frac{\partial}{\partial \rho}+\frac{1-\mu^{2}}{\rho} \frac{\partial}{\partial \mu}\right)\right] \chi
\end{aligned}
$$

(ii) For the components of stress,

$$
\begin{aligned}
\sigma_{\rho}= & {\left[(2-v) \mu \frac{\partial}{\partial \rho}+\frac{v\left(1-\mu^{2}\right)}{\rho} \frac{\partial}{\partial \mu}\right] \nabla^{2} \chi } \\
- & \frac{\partial^{2}}{\partial \rho^{2}}\left(\mu \frac{\partial}{\partial \rho}+\frac{1-\mu^{2}}{\rho} \frac{\partial}{\partial \mu}\right) \chi \\
\sigma_{\phi}= & -(1-v)\left(\mu \frac{\partial}{\partial \rho}-\frac{1-\mu^{2}}{\rho} \frac{\partial}{\partial \mu}\right) \nabla^{2} \chi \\
& +\left(\frac{\partial^{2}}{\partial \rho^{2}}+\frac{1}{\rho} \frac{\partial}{\partial \rho}-\frac{\mu}{\rho^{2}} \frac{\partial}{\partial \mu}\right) \\
& \times\left(\mu \frac{\partial}{\partial \rho}+\frac{1-\mu^{2}}{\rho} \frac{\partial}{\partial \mu}\right) \chi,
\end{aligned}
$$




$$
\begin{gathered}
\sigma_{\theta}=\left(v \nabla^{2}-\frac{1}{\rho} \frac{\partial}{\partial \rho}+\frac{\mu}{\rho^{2}} \frac{\partial}{\partial \mu}\right)\left(\mu \frac{\partial}{\partial \rho}+\frac{1-\mu^{2}}{\rho} \frac{\partial}{\partial \mu}\right) \chi \\
\sigma_{\rho \phi}=\left(1-\mu^{2}\right)^{1 / 2}\left[-(1-v)\left(\frac{\partial}{\partial \rho}+\frac{\mu}{\rho} \frac{\partial}{\partial \mu}\right) \nabla^{2} \chi\right. \\
\left.+\frac{\partial}{\partial \rho}\left(\frac{1}{\rho} \frac{\partial}{\partial \mu}\right)\left(\mu \frac{\partial}{\partial \rho}+\frac{1-\mu^{2}}{\rho} \frac{\partial}{\partial \mu}\right) \chi\right] ;
\end{gathered}
$$

(iii) For the components of strain,

$$
\begin{aligned}
& \epsilon_{\rho}=\frac{\partial \mu_{\rho}}{\partial \rho}, \\
& \epsilon_{\phi}=\frac{u_{\rho}}{\rho}-\frac{\left(1-\mu^{2}\right)^{1 / 2}}{\rho} \frac{\partial u_{\phi}}{\partial \mu}, \\
& \epsilon_{\theta}=\frac{u_{\rho}}{\rho}+\frac{u_{\phi}}{\rho} \cot \phi, \\
& \epsilon_{\rho \phi}=\frac{\partial u_{\phi}}{\partial \rho}-\frac{u_{\phi}}{\rho}-\frac{\left(1-\mu^{2}\right)^{1 / 2}}{\rho} \frac{\partial u_{\rho}}{\partial \mu} .
\end{aligned}
$$

The other components vanish identically. The expressions for $\sigma_{\phi}$ and $\sigma_{\theta}$ are here modified slightly for simplicity. $v$ is the Poisson ratio and $G$ the modulus of rigidity. $\nabla^{2}$ is a Laplace operator. For the axisymmetric case, it is given in spherical coordinates by

$$
\nabla^{2}=\frac{1}{\rho^{2}} \frac{\partial}{\partial \rho}\left(\rho^{2} \frac{\partial}{\partial \rho}\right)+\frac{1}{\rho^{2}} \frac{\partial}{\partial \mu}\left[\left(1-\mu^{2}\right) \frac{\partial}{\partial \mu}\right] .
$$

\section{A CENTER OF DILATATION} by

Define a second set of spherical coordinates $\left(\rho^{*}, \phi^{*}, \theta\right)$

$$
\begin{aligned}
& x=\rho^{*} \sin \phi^{*} \cos \theta, \\
& y=\rho^{*} \sin \phi^{*} \sin \theta, \\
& z-c=\rho^{*} \cos \phi^{*} .
\end{aligned}
$$

This set of spherical coordinates has its pole on the $z$ axis at the point $0^{*}$ or $z=c$. Similarly, let

$$
\mu^{*}=\cos \phi^{*} \text {. }
$$

Consider a center of dilatation of strength $s$ located on the $z$ axis at the point $0^{*}$ inside a sphere $\rho \leqslant a$. Suppose that the Love stress function in question is composed of two parts as follows:

$$
\chi=\chi_{0}+\chi_{1} \text {. }
$$

The first part is the Love stress function for the center of dilatation in an infinite solid. The second part is added to annul the residual tractions on the surface of the sphere left by the first part. We construct

$$
\begin{aligned}
& \chi_{0}=2 G s Q_{0}\left(\mu^{*}\right), \\
& \chi_{1}=\sum_{n=1}^{\infty} A_{n} \rho^{n} P_{n}(\mu)+\sum_{n=0}^{\infty} B_{n+2} \rho^{n+2} P_{n}(\mu),
\end{aligned}
$$

where $Q_{0}$ is a Legendre function of the second kind of order zero and $P_{n}$ a Legendre function of the first kind of degree $n$. $A_{n}$ and $B_{n}$ are parametric coefficients. Both parts are biharmonic.

From the first part $\chi_{0}$, we find the following radial components of displacement and stress by Eqs. (3) and (4), respectively, referred to $\rho^{*}$ and $\mu^{*}$ :

$$
\mu_{\rho^{*}}=\frac{s}{\rho^{* 2}}, \quad \sigma_{\rho^{*}}=-\frac{4 G s}{\rho^{* 3}} .
$$

With proper choice of units, the first relation defines $s$ or the strength of the center of dilatation.

When $\rho>|c|, \chi_{0}$ can be expanded into the series:

$$
\chi_{0}=2 G s\left[Q_{0}(\mu)-\sum_{n=0}^{\infty} \frac{c^{n+1}}{(n+1) \rho^{n+1}} P_{n}(\mu)\right] .
$$

The following normal and tangential components of stress are derived from $\chi$, or the sum of $\chi_{0}$ and $\chi_{1}$, for $\rho>|c|$ :

$$
\begin{aligned}
\sigma_{\rho}= & -\frac{4 G s}{\rho^{3}}-\sum_{n=0}^{\infty}\left\{\frac{2 G s(n+1)(n+2) c^{n}}{\rho^{n+3}}\right. \\
& +\frac{2 n+5}{2 n+3}(n+1)\left(n^{2}-n-2-2 v\right) B_{n+3} \rho^{n} \\
& +n(n-1)\left[(n+1) A_{n+1}\right. \\
& \left.\left.-\frac{2(3 n-4 n v+2-2 v)}{2 n-1} B_{n+1}\right] \rho^{n-2}\right\} P_{n}(\mu), \\
\sigma_{\rho \phi}= & \left(1-\mu^{2}\right)^{1 / 2} \sum_{n=1}^{\infty}\left\{-\frac{2 G s(n+2) c^{n}}{\rho^{n+3}}+\frac{2 n+5}{2 n+3}\right. \\
& \times\left(n^{2}+2 n-1+2 v\right) B_{n+3} \rho^{n} \\
& +(n-1)\left[(n+1) A_{n+1}-\frac{2(3 n-4 n v+2-2 v)}{2 n-1}\right. \\
& \left.\left.\times B_{n+1}\right] \rho^{n-2}\right\} P_{n}^{\prime}(\mu) .
\end{aligned}
$$

The prime on $P_{n}$ denotes a derivative. They are found with the aid of the following relations:

$$
\begin{aligned}
& \frac{\partial}{\partial z}=\mu \frac{\partial}{\partial \rho}+\frac{1-\mu^{2}}{\rho} \frac{\partial}{\partial \mu}, \\
& \frac{\partial}{\partial z} Q_{0}(\mu)=\frac{1}{\rho}, \\
& \frac{\partial}{\partial z}\left[\rho^{n} P_{n}(\mu)\right]=n \rho^{n-1} P_{n-1}(\mu), \quad(n \geqslant 1), \\
& \frac{\partial}{\partial z}\left(\frac{P_{n}(\mu)}{\rho^{n+1}}\right)=-(n+1) \frac{P_{n+1}(\mu)}{\rho^{n+2}}, \quad(n \geqslant 0),
\end{aligned}
$$

and also the recurrence formulas ${ }^{7}$ for $P_{n}$. Consequently, the normal and tangential tractions on the surface of the sphere are annulled if we substitute $\rho=a$ into Eq. (13) and equate each coefficient of $P_{n}(\mu)$ as well as of $\left(1-\mu^{2}\right)^{1 / 2} P_{n}^{\prime}(\mu)$ to zero. This leads to two sets of equations, of which the solution is

$$
\begin{aligned}
B_{3}= & \frac{12 G s}{5(1+v) a^{3}}, \\
B_{n+3}= & \frac{G s(n+2)(2 n+1)(2 n+3) c^{n}}{(2 n+5)\left(n^{2}+n+2 n v+1+v\right) a^{2 n+3}}, \quad(n \geqslant 1), \\
A_{3}= & \frac{16 G s(4-5 v)}{15(1+v) a^{3}}-\frac{28 G s c^{2}}{(7+5 v) a^{5}}, \\
A_{n+1}= & \frac{2 G s n(2 n-3)(3 n-4 n v+2-2 v) c^{n-2}}{(n+1)(2 n+1)\left(n^{2}-3 n+2 n v+3-3 v\right) a^{2 n-1}} \\
& \quad-\frac{G s(n+2)(2 n+3) c^{n}}{\left(n^{2}+n+2 n v+1+v\right) a^{2 n+1}}, \quad(\mathrm{n} \geqslant 3) .
\end{aligned}
$$


The preceding solution does not include $A_{1}, A_{2}$, and $B_{2}$. The term involving $A_{1}$ produces no effect on both displacement and stress and may therefore be omitted. The terms involving $A_{2}$ and $B_{2}$ produce no effect on stress but each gives a rigid body displacement in the $z$ direction of amounts $-A_{2} /$
$G$ and $B_{2}(5-6 v) / G$, respectively. If the rigid body displacement of the sphere is zero, these terms are absent.

The components of displacement and stress are given below:

$$
\begin{aligned}
u_{\rho}= & \frac{s(\rho-c \mu)}{\rho^{* 3}}-\frac{1}{2 G} \sum_{n=0}^{\infty} \frac{2 n-5}{2 n+3}(n+1)(n-2+4 v) B_{n+3} \rho^{n+1} P_{n}(\mu) \\
& -\frac{1}{2 G} \sum_{n=2}^{\infty} n\left[(n+1) A_{n+1}-\frac{2(3 n-4 n v+2-2 v)}{2 n-1} B_{n+1}\right] \rho^{n-1} P_{n}(\mu), \\
u_{\phi}= & \frac{s c\left(1-\mu^{2}\right)^{1 / 2}}{\rho^{* 3}}+\frac{1}{2 G}\left(1-\mu^{2}\right)^{1 / 2} \sum_{n=1}^{\infty} \frac{2 n-5}{2 n+3}(n+5-4 v) B_{n+3} \rho^{n+1} P_{n}^{\prime}(\mu) \\
& +\frac{1}{2 G}\left(1-\mu^{2}\right)^{1 / 2} \sum_{n=2}^{\infty}\left[(n+1) A_{n+1}-\frac{2(3 n-4 n v+2-2 v)}{2 n-1} B_{n+1}\right] \rho^{n-1} P_{n}^{\prime}(\mu),
\end{aligned}
$$

and

$$
\begin{aligned}
\sigma_{p}= & G s\left(\frac{1}{\rho^{* 3}}-\frac{3(\rho-c \mu)^{2}}{\rho^{* 5}}\right)-\sum_{n=0}^{\infty}\left\{\frac{2 n+5}{2 n+3}(n+1)\left(n^{2}-n-2-2 v\right) B_{n+3} \rho^{n}\right. \\
& \left.+n(n-1)\left[(n+1) A_{n+1}-\frac{2(3 n-4 n v+2-2 v)}{2 n-1} B_{n+1}\right] \rho^{n-2}\right\} P_{n}(\mu), \\
\sigma_{\phi}= & -2 G s\left(\frac{2}{\rho^{* 3}}-\frac{3(\rho-c \mu)^{2}}{\rho^{* 5}}\right)+\sum_{n=2}^{\infty}(n+1) A_{n+1} \rho^{n-2}\left[n(n-1) P_{n}(\mu)-P_{n-1}^{\prime}(\mu)\right] \\
& +\sum_{n=0}^{\infty} B_{n+3} \rho^{n}\left\{(1-v)(2 n+2)(2 n+5)\left[P_{n}(\mu)-2 \mu P_{n-1}(\mu)\right]\right. \\
& \left.+(n+1)(n+2)\left[(n+2) P_{n}(\mu)+2 \mu P_{n+1}(\mu)\right]-(n+3) \mu P_{n}^{\prime}(\mu)\right\}, \\
\sigma_{\theta}= & \frac{2 G s}{\rho^{* 3}}+\sum_{n=2}^{\infty}(n+1) A_{n+1} \rho^{n-2} P_{n-1}^{\prime}(\mu)+\sum_{n=0}^{\infty} B_{n+3} \rho^{n}\{[2 v(2 n+5) \\
& \left.-(n+2)](n+1) P_{n}(\mu)+(n+3) \mu P_{n}^{\prime}(\mu)\right\} \\
\sigma_{\rho \phi}= & -\frac{6 G s c\left(1-\mu^{2}\right)^{1 / 2}(\rho-c \mu)}{\rho^{* 5}}+\left(1-\mu^{2}\right)^{1 / 2} \sum_{n=1}^{\infty}\left\{\frac{2 n+5}{2 n+3}\left(n^{2}+2 n-1+2 v\right) B_{n+3} \rho^{n}\right. \\
& \left.+(n+1)\left[(n+1) A_{n+1}-\frac{2(3 n-4 n v+2-2 v)}{2 n-1} B_{n+1}\right] \rho^{n-2}\right\} P_{n}^{\prime}(\mu),
\end{aligned}
$$

where

$$
\rho^{*}=\left(\rho^{2}+c^{2}-2 c \rho \mu\right)^{1 / 2} .
$$

The sphere is assumed to undergo no rigid body displacement. For the sake of brevity, the components of strain are not shown. They can be found readily from Eq. (5) whenever needed.

\section{A DOUBLET}

Consider a doublet located on the $z$ axis at the point $0^{*}$ or $z=c$ inside a sphere $\rho \geqslant a$. Let the doublet be oriented axisymmetrically in the $z$ direction. Likewise, suppose that the required Love stress function is composed of two parts as follows:

$$
\chi=\chi_{0}+\chi_{1}
$$

To find the fitst part $\chi_{0}$, consider a Love stress function in the form

$$
\chi_{0}^{*}=K \rho^{*},
$$

where $K$ is a constant. This function is biharmonic and gives the following radial components of displacement and stress referred to $\rho^{*}$ and $\mu^{*}$ :

$$
u_{\rho^{*}}=\frac{2 K(1-v) \mu^{*}}{G \rho^{*}}, \quad \sigma_{\rho^{*}}=-\frac{2 K(2-v) \mu^{*}}{\rho^{* 2}}
$$

It gives a concentrated force $P$ in the $z$ direction at the point $0^{*}$ of amount ${ }^{8}$

$$
P=8 \pi(1-v) K
$$

Let a concentrated force $P / h$ be applied at the point $0^{*}$ or $z=c$ in the $z$ direction and an equal and opposite concentrated force be applied at the point $z=c-h$ on the $z$ axis. When $h$ tends to zero while the concentrated force $P$ remains constant, we obtain a doublet at the point $0^{*}$ oriented in the $z$ direction. The corresponding Love stress function is

$$
\begin{aligned}
\chi_{0}= & \lim _{h \rightarrow 0}\left\{\frac{K}{h}\left[\rho^{2} \sin ^{2} \phi+(z-c)^{2}\right]^{1 / 2}\right. \\
& \left.-\frac{K}{h}\left[\rho^{2} \sin ^{2} \phi+(z-c+h)^{2}\right]^{1 / 2}\right\} \\
= & -K \mu^{*},
\end{aligned}
$$

which gives the following radial components of displacement and stress referred to $\rho^{*}$ and $\mu^{*}$ : 


$$
\begin{aligned}
& u_{\rho^{*}}=-\frac{K}{2 G \rho^{* 2}}\left[1-(5-4 v) \mu^{* 2}\right], \\
& \sigma_{\rho^{*}}=\frac{2 K}{\rho^{* 3}}\left[1+v-(5-v) \mu^{* 2}\right] .
\end{aligned}
$$

Suppose that three mutually orthogonal doublets each of strength $s$ are combined to form a center of dilatation of strength $s$. It can be shown that

$$
K=G s /(1-2 v)
$$

The first part $\chi_{0}$ in Eq. (23) can be expanded for $\rho>|c|$ into the series:

$$
\begin{aligned}
\chi_{0}= & -K \mu-K \sum_{n=2}^{\infty} \frac{n c^{n-1}}{(2 n-1) \rho^{n-1}} P_{n}(\mu) \\
& +K \sum_{n=0}^{\infty} \frac{(n+2) c^{n+1}}{(2 n+3) \rho^{n+1}} P_{n}(\mu),
\end{aligned}
$$

from which the following normal and tangential components of stress are found:

$$
\begin{aligned}
\sigma_{\rho}= & -\frac{4 K(1-2 v)}{3 \rho^{3}}-\frac{12 K c(1-4 v) \mu}{5 \rho^{4}} \\
& +K \sum_{n=2}^{\infty}\left(\frac{(n+1)^{2}(n+2)(n-2+4 v) c^{2}}{(2 n+3) \rho^{2}}\right. \\
& \left.-\frac{n(n-1)\left(n^{2}+3 n-2 v\right)}{2 n-1}\right) \frac{c^{n-2}}{\rho^{n+1}} P_{n}(\mu), \\
\sigma_{\rho}= & -K\left(1-\mu^{2}\right)^{1 / 2}\left[\frac{6(1-4 v) c}{5 \rho^{4}}\right. \\
& +\sum_{n=2}^{\infty}\left(-\frac{(n+1)(n+2)(n-2+4 v) c^{2}}{(2 n+3) \rho^{2}}\right. \\
& \left.\left.+\frac{(n-1)\left(n^{2}-2+2 v\right)}{2 n-1}\right) \frac{c^{n-2}}{\rho^{n+1}} P_{n}^{\prime}(\mu)\right] .
\end{aligned}
$$

The second part $\chi_{1}$ is constructed as follows:

$$
\chi_{1}=\sum_{n=3}^{\infty} A_{n} \rho^{n} P_{n}(\mu)+\sum_{n=1}^{\infty} B_{n+2} \rho^{n+2} P_{n}(\mu),
$$

which is the same as in Eq. (10), except that the terms involving $A_{1}, A_{2}$, and $B_{2}$ are now absent. This implies that the sphere undergoes no rigid body displacement. The components of stress derived from $\chi_{1}$ are the same as the corresponding parts in Eq. (13). The residual normal and tangential tractions on the surface of sphere are therefore annulled if we substitute $\rho=a$ into the expressions for $\sigma_{\rho}$ and $\sigma_{\rho \phi}$ derived from $\chi$, or the sum of $\chi_{0}$ and $\chi_{1}$, and equate each coefficient of $P_{n}(\mu)$ as well as of $\left(1-\mu^{2}\right)^{1 / 2} P_{n}^{\prime}(\mu)$ to zero. Likewise, two sets of equations are obtained. Their solution is

$$
\begin{aligned}
& B_{3}=\frac{2 K(1-2 v)}{5(1+v) a^{3}}, \\
& B_{4}=\frac{3 K(1-4 v) c}{7(1+v) a^{5}},
\end{aligned}
$$

$$
\begin{aligned}
B_{n+3}= & \frac{(n+2) K}{2(2 n+5)}\left(\frac{n(n-1)(2 n+3)}{n^{2}+n+2 n v+1+v}\right. \\
& \left.-\frac{(n+1)(2 n+1)(n-2+4 v) c^{2}}{\left(n^{2}+n+2 n v+1+v\right) a^{2}}\right) \\
& \times \frac{c^{n-2}}{a^{2 n+1}}, \quad(n \geqslant 2) \\
A_{n+1}= & \frac{2(3 n-4 n v+2-2 v)}{(n+1)(2 n-1)} B_{n+1}-\frac{2 n+5}{2 n+1} B_{n+3} a^{2} \\
& -\frac{2 K\left(n^{2}+n-2 n v+1-v\right) c^{n-2}}{(n+1)(2 n-1)(2 n+1) a^{2 n-1}}, \quad(n \geqslant 2) .
\end{aligned}
$$

The following components of displacement and stress are found from the first part $\chi_{0}$ :

$$
\begin{aligned}
& u_{\rho}=\frac{K}{G}\left(\frac{2(1-v)(\rho \mu-c) \mu+\rho\left(1-\mu^{2}\right)}{\rho^{* 3}}\right. \\
& \left.-\frac{3 \rho^{2}(\rho-c \mu)\left(1-\mu^{2}\right)}{\rho^{* 5}}\right) \\
& u_{\phi}=-\frac{K}{G}\left(1-\mu^{2}\right)^{1 / 2}\left(\frac{2(1-2 v)(\rho \mu-c)-\rho \mu}{\rho^{* 3}}\right. \\
& \left.-\frac{3 c \rho^{2}\left(1-\mu^{2}\right)}{2 \rho^{* 5}}\right) \\
& \sigma_{\rho}=\frac{2 K}{\rho^{* 3}}\left[1+v+(1-2 v) \mu^{2}\right] \\
& -\frac{3 K}{\rho^{* 5}}\left\{\begin{array}{l}
2(2-v)(\rho \mu-c)(\rho-c \mu) \mu \\
+\left[5 \rho^{2}-2(2+v) c \rho \mu+2 v c^{2}\right]\left(1-\mu^{2}\right)
\end{array}\right\} \\
& +\frac{15 K \rho^{2}(\rho-c \mu)^{2}\left(1-\mu^{2}\right)}{\rho^{* 7}} \\
& \sigma_{\phi}=-\frac{2 K(1-2 v) \mu^{2}}{\rho^{* 3}}+\frac{6 K}{\rho^{* 5}} \\
& \times\left[\begin{array}{l}
(1-v)(\rho \mu-c)\left(\rho \mu-2 c \mu^{2}+c\right) \\
+\rho(3 \rho-2 c \mu)\left(1-\mu^{2}\right)
\end{array}\right] \\
& -\frac{15 K \rho^{2}(\rho-c \mu)^{2}\left(1-\mu^{2}\right)}{\rho^{* 7}} \text {, } \\
& \sigma_{\theta}=\frac{2 K(1+v)}{\rho^{* 3}}-\frac{3 K}{\rho^{* 5}}\left[\begin{array}{l}
2 v(\rho \mu-c)^{2} \\
+\left(3 \rho^{2}-3 c \rho \mu-c \mu\right)\left(1-\mu^{2}\right)
\end{array}\right], \\
& \sigma_{\rho \phi}=K\left(1-\mu^{2}\right)^{1 / 2}\left\{\frac{2 v}{\rho^{* 3}}+\frac{6}{\rho^{* 5}}\right. \\
& \times\left[\begin{array}{c}
(1-v)(\rho \mu-c)(\rho-2 c \mu) \\
-\rho\left(\rho \mu+c-2 c \mu^{2}\right)
\end{array}\right] \\
& \left.+\frac{15 c \rho^{2}(\rho-c \mu)\left(1-\mu^{2}\right)}{\rho^{* 7}}\right\}
\end{aligned}
$$

where $\rho^{*}$ is given before in Eq. (18). The components of displacement and stress from the second part $\chi_{1}$ are the same as the corresponding parts in Eqs. (16) and (17). The components of strain are given by Eq. (5).

THE PARTICULAR CASE $c=0$

In the particular case $c=0$, the point defect is located at the center of the sphere. The solutions are considerably simplified. For a center of dilatation of strength $s$, 
TABLE I. $\mu_{\rho} / s$ and $\mu_{\phi} / s$ on $\rho=a$ due to a center of dilatation for $a=2$ and $v=\frac{1}{4}$.

\begin{tabular}{|c|c|c|c|c|c|c|c|c|}
\hline \multirow[b]{2}{*}{$\phi$} & \multicolumn{4}{|c|}{ Values of $\mu_{\rho} / s$} & \multicolumn{4}{|c|}{ Values of $\mu_{\phi} / s$} \\
\hline & $c=0$ & $c=\frac{1}{4}$ & $c=\frac{1}{2}$ & $c=\frac{3}{4}$ & $c=0$ & $c=\frac{1}{4}$ & $c=\frac{1}{2}$ & $c=\frac{3}{4}$ \\
\hline $0^{\circ}$ & -0.1500 & -0.0218 & 0.3127 & 1.0679 & 0 & 0 & 0 & 0 \\
\hline 15 & -0.1500 & -0.0321 & 0.2550 & 0.8413 & 0 & -0.0307 & -0.1193 & -0.3275 \\
\hline 30 & -0.1500 & -0.0593 & 0.1153 & 0.3708 & 0 & -0.0544 & -0.1951 & -0.4795 \\
\hline 45 & -0.1500 & -0.0952 & -0.0386 & -0.0333 & 0 & -0.0669 & -0.2122 & -0.4516 \\
\hline 60 & -0.1500 & -0.1304 & -0.1571 & -0.2633 & 0 & -0.0677 & -0.1831 & -0.3313 \\
\hline 75 & -0.1500 & -0.1583 & -0.2242 & -0.3481 & 0 & -0.0594 & -0.1305 & -0.1922 \\
\hline 90 & -0.1500 & -0.1759 & -0.2462 & -0.3428 & 0 & -0.0460 & -0.0743 & -0.0725 \\
\hline 105 & -0.1500 & -0.1836 & -0.2370 & -0.2908 & 0 & -0.0314 & -0.0271 & 0.0131 \\
\hline 120 & -0.1500 & -0.1838 & -0.2108 & -0.2208 & 0 & -0.0186 & 0.0052 & 0.0622 \\
\hline 135 & -0.1500 & -0.1797 & -0.1792 & -0.1514 & 0 & -0.0092 & 0.0213 & 0.0781 \\
\hline 150 & -0.1500 & -0.1743 & -0.1508 & -0.0945 & 0 & -0.0035 & 0.0230 & 0.0675 \\
\hline 165 & -0.1500 & -0.1700 & -0.1316 & -0.0575 & 0 & -0.0009 & 0.0143 & 0.0385 \\
\hline 180 & -0.1500 & -0.1684 & -0.1248 & -0.0448 & 0 & 0 & 0 & 0 \\
\hline
\end{tabular}

TABLE II. $\mu_{\rho} / s$ and $\mu_{\phi} / s$ on $\rho=a$ due to a doublet for $a=2$ and $v=\frac{1}{4}$.

\begin{tabular}{|c|c|c|c|c|c|c|c|c|}
\hline \multirow[b]{2}{*}{$\phi$} & \multicolumn{4}{|c|}{ Values of $\mu_{p} / s$} & \multicolumn{4}{|c|}{ Values of $\mu_{\phi} / s$} \\
\hline & $c=0$ & $c=\frac{1}{4}$ & $c=\frac{1}{2}$ & $c=\frac{3}{4}$ & $c=0$ & $c=\frac{1}{4}$ & $c=\frac{1}{2}$ & $c=\frac{3}{4}$ \\
\hline $0^{\circ}$ & 2.1253 & 3.0267 & 4.4928 & 7.0582 & 0 & 0 & 0 & 0 \\
\hline 15 & 1.8631 & 2.5186 & 3.4319 & 4.6450 & -0.5862 & -0.8582 & -1.2982 & -2.0291 \\
\hline 30 & 1.1470 & 1.2495 & 1.1454 & 0.5735 & -1.0153 & -1.3479 & -1.7384 & -2.0758 \\
\hline 45 & 0.1688 & -0.2021 & -0.8290 & 1.6626 & -1.1723 & -1.3160 & -1.3017 & -0.9819 \\
\hline 60 & -0.8094 & -0.3018 & -1.7836 & 2.0789 & -1.0153 & -0.8630 & -0.5060 & 0.0271 \\
\hline 75 & -1.5255 & -1.7906 & -1.8511 & -1.6820 & -0.5862 & -0.2215 & 0.2123 & 0.6063 \\
\hline 90 & -1.7876 & -1.6846 & -1.4163 & -1.0772 & 0 & 0.3848 & 0.6807 & 0.8399 \\
\hline 105 & -1.5255 & -1.1557 & -0.7897 & -0.4958 & 0.5862 & 0.8135 & 0.8952 & 0.8648 \\
\hline 120 & -0.8094 & -0.4171 & 0.1553 & -0.0063 & 1.0153 & 1.0089 & 0.9104 & 0.7741 \\
\hline 135 & 0.1688 & 0.3418 & 0.3918 & 0.3760 & 1.1723 & 0.9771 & 0.7855 & 0.6197 \\
\hline 150 & 1.1470 & 0.9779 & 0.8042 & 0.6493 & 1.0153 & 0.7591 & 0.5689 & 0.4295 \\
\hline 165 & 1.8631 & 1.3954 & 1.0588 & 0.8135 & 0.5862 & 0.4122 & 0.2973 & 0.2193 \\
\hline 180 & 2.1253 & 1.5403 & 1.1447 & 0.8682 & 0 & 0 & 0 & 0 \\
\hline
\end{tabular}

$$
\chi=2 G s Q_{0}(\mu)+A_{3} \rho^{3} P_{3}(\mu)+B_{3} \rho^{3} P_{1}(\mu),
$$
where

$$
A_{3}=\frac{16(4-5 v) G s}{15(1+v) a^{3}}, \quad B_{3}=\frac{12 G s}{5(1+v) a^{3}} .
$$

For a doublet of strength $s$ oriented axisymmetrically about the $z$ axis,

$$
\begin{aligned}
\chi= & -\frac{G s \mu}{1-2 v}+A_{3} \rho^{3} P_{3}(\mu)+A_{5} \rho^{5} P_{5}(\mu) \\
& +B_{3} \rho^{3} P_{1}(\mu)+B_{5} \rho^{5} P_{3}(\mu)
\end{aligned}
$$

where

$$
\begin{aligned}
& A_{3}=\frac{2 G s}{45 a^{3}}\left(\frac{4(4-5 v)}{1+v}-\frac{25\left(7-v^{2}\right)}{(1-2 v)(7+5 v)}\right), \\
& A_{5}=\frac{16(7-9 v) G s}{45(1-2 v)(7+5 v) a^{5}} \\
& B_{3}=\frac{2 G s}{5(1+v) a^{3}} \\
& B_{5}=\frac{28 G s}{9(1-2 v)(7+5 v) a^{5}} .
\end{aligned}
$$

In either case, the rigid body displacement is assumed to be zero.

\section{NUMERICAL EXAMPLES}

The components of displacement on the surface of the sphere $\rho=a$ are computed in both cases for the following values:

$$
a=2, \quad v=\frac{1}{4}, \quad c=0\left(\frac{1}{4}\right) \frac{3}{4} .
$$

The sphere is assumed to undergo no rigid body displacement. The results are shown in Tables I and II.

\section{ACKNOWLEDGMENTS}

The authors wish to acknowledge VPI \& SU sponsorship in support of National Science Foundation Grant No. DMR-8000933.

${ }^{\prime} H$. Ekstein, Phys. Rev. 68, 120 (1945).

${ }^{2}$ K. Huang, Proc. R. Soc. London Ser. A 190, 102 (1947).

${ }^{3}$ D. Eshelby, J. Appl. Phys. 25, 255 (1954).

${ }^{4} \mathrm{~J}$. Dundurs and D. L. Guell, Developments in Theoretical and Applied Mechanics (Pergamon, New York, 1965), Vol. II.

${ }^{5}$ A. E. H. Love, Mathematical Theory of Elasticity (Dover, New York, 1944), 4th edition.

${ }^{6}$ C. B. Ling and K. L. Yang, J. Appl. Mech. 18, A367 (1951).

${ }^{7}$ T. M.MacRobert, Spherical Harmonics (Pergamon, New York, 1967), 3rd edition.

${ }^{8} \mathrm{~S}$. Timoshenko and J. N. Goodier, Theory of Elasticity (McGraw-Hill, New York, 1951), 2nd edition. 